\title{
MARINOS EN TIERRA: \\ INSERCIÓN SOCIAL DE LA OFICIALIDAD NAVAL EN LA CIUDAD DE CARTAGENA (SIGLOS XVIII-XIX)
}

\author{
Pablo Ortega-del-Cerro* \\ Instituto de Ciências Sociais da Universidade de Lisboa
}

\begin{abstract}
RESUMEN
El objetivo del presente artículo es estudiar la forma en que los oficiales navales se insertaron socialmente en la ciudad de Cartagena -una de las bases más importantes de la Armada española y capital del Departamento Marítimo del Mediterráneo- a lo largo de los siglos XVIII y XIX. Tomamos como punto de partida que estos oficiales fueron, además de militares, seres sociales que vivieron su cotidianeidad en ciudades donde la Armada tenía un establecimiento, es decir, eran individuos que formaban parte de redes sociales de alcance local, cuyas pautas de sociabilidad estaban determinadas, en gran medida, por las características castrenses de la urbe. Se trata, en definitiva, de conocer qué posición tuvieron estos oficiales en la estructura social cartagenera y cómo se insertaban en la vida social local a lo largo del setecientos y ochocientos.
\end{abstract}

PALABRAS CLAVE: oficiales navales, integración social, Cartagena, siglo XVIII, siglo XIX

Enviado: $15-12-2019$

Aceptado: $10-2-2020$

*pablo_odc@hotmail.com

(C) Baetica. Estudios Historia Moderna y Contemporánea, 39, 2019, 321-348. Facultad de Filosofía y Letras, Universidad de Málaga. Departamento de Historia Moderna y Contemporánea 


\title{
DISEMBARKED NAVAL OFFICERS: THE SPANISH NAVY OFFICERS AND THEIR SOCIAL INSERTION IN THE CITY OF CARTAGENA (18TH-19TH CENTURIES)
}

\author{
Pablo Ortega-del-Cerro* \\ Instituto de Ciências Sociais da Universidade de Lisboa
}

\begin{abstract}
The objective of this article is to study the social integration of the naval officers in the city of Cartagena - one of the most important bases of the Spanish Navy and the capital of the Maritime Department of the Mediterranean- throughout the eighteenth and nineteenth centuries. The starting point is that these officers were social beings who lived their daily lives in cities where the Navy had a base, that is, they were individuals who were part of social networks of local reach, whose patterns of sociability were determined largely due to the military characteristics of the city. In shorth, this work seeks to examine the position of these officers in the Cartagena social structure and how they were inserted in the local social life throughout the eighteenth and nineteenth centuries.
\end{abstract}

KEYWORDS: naval officers, social insertion, Cartagena, 18th century, 19th century

*pablo_odc@hotmail.com 


\section{INTRODUCCIÓN ${ }^{1}$}

La visión más extendida que tenemos actualmente de la oficialidad de la Armada española se focaliza en tres aspectos. En primer lugar, destaca la representación de los oficiales navales como grandes científicos del siglo de las Luces y precursores de adelantos técnicos; segundo, como exploradores y responsables de complejas expediciones alrededor del globo; y, por último, como relevantes militares que protagonizaron famosas batallas navales ${ }^{2}$. Sin negar la relevancia e importancia de todas estas facetas, la oficialidad fue un grupo socio-profesional de individuos que tuvo otros muchos rasgos y roles. Además de surcar los mares, descubrir nuevos territorios y defender a la monarquía en los océanos, eran sujetos que compartían con el resto de la sociedad problemas, necesidades, patrones de comportamiento y cosmovisiones. El objetivo de este trabajo es, precisamente, analizar y comprender la integración de la oficialidad naval en la sociedad de su tiempo, conocer cómo estos oficiales eran parte de la estructura social, cómo se insertaban en los recovecos de la vida social y cómo se comportaban en la cotidianeidad.

Este objetivo queda acotado por dos coordenadas básicas, tiempo y espacio. La cronología escogida abarca desde comienzos del siglo XVIII, especialmente desde mediados de esta centuria, hasta finales del XIX. Se trata, indudablemente, de un tiempo sugerente y complejo. Durante estos dos siglos emergieron mutaciones que acabaron por transformar profundamente el orden político y el panorama social. Los oficiales de la Armada fueron testigos privilegiados de estos cambios y sus experiencias constituyen un material de gran interés para comprender el desarrollo de la sociedad. La variable espacial queda circunscrita a la ciudad de Cartagena -usualmente denominada como Cartagena de Levante para no confundirla con Cartagena de Indias-, situada en el sureste español. Junto a Cádiz/Isla de León, Ferrol y La Habana, Cartagena era una de las grandes sedes que tuvo la Real Armada desde el siglo XVIII y, por tanto, se convirtió en una de las ciudades navales por excelencia de la Monarquía. Este hecho permite en-

1. Este trabajo se ha realizado en el marco del Proyecto de Investigación "Entornos sociales de cambio. Nuevas solidaridades y ruptura de jerarquías (siglos XVI-XX)", HAR2017-84226-C6-1-P, Ministerio de Ciencia, Innovación y Universidades; y el Programa de Formación Postdoctoral en el extranjero, Fundación Séneca-Agencia de Ciencia y Tecnología de la Región de Murcia, 20703/PD/18.

2. La lista de referencias podría ser amplísima. H. O’Donnell (2002); M. Gil MuÑoz (2015); M. García Garralón (2018). 
tender que la oficialidad naval tuviera una muy relevante posición dentro de su estructura social. No obstante, el carácter de este privilegiado estatus dependió mucho de los rasgos e idiosincrasia de la sociedad local, de tal forma que los oficiales tuvieron formas de integración muy diferentes en cada una de estas ciudades ${ }^{3}$.

La pregunta que deseamos resolver en este trabajo, por tanto, es cómo los oficiales de la Armada se integraron en la sociedad cartagenera de los siglos XVIII y XIX, cómo vivieron estos individuos su cotidianeidad social en esta ciudad del Levante español. Es indudable que estas preguntas abren la puerta a multitud de temáticas y problemas de amplio calado y enfoque diverso, y por ello se ha optado por el análisis de un extenso abanico de fuentes de diferente naturaleza que permitan examinar las diversas caras de este poliédrico problema. Destaca la documentación encontrada en el Archivo Municipal de Cartagena, el Archivo Naval de Cartagena y el Archivo Histórico Provincial de Murcia, aunque hay referencia a otros centros documentales. La variedad de aspectos que van a ser tratados permitirá ofrecer una visión de conjunto, tanto de la inserción estructural de la oficialidad en la sociedad local, como de las transformaciones que se fueron dando a lo largo de estos dos siglos.

\section{ASENTAMIENTO DE LA REALARMADA EN EL DEPARTAMENTO DEL MEDITERRÁNEO}

En 1503 la reina Isabel recuperaba las tierras del señorío de Cartagena, que habían sido cedidas a Pedro Fajardo en 1464 por Enrique IV. Con esta medida se pretendía incorporar a las tierras de realengo un enclave que sirviera de puerto mercante y militar para la Corona de Castilla en el Mediterráneo y el Levante. Con una configuración geográfica excepcional - una bahía cerrada y resguardada por un sistema montañoso que favorece la defensa natural-, su papel militar se potenció a lo largo del siglo XVI ${ }^{4}$. Se convirtió en base de las galeras reales y punto logístico y militar excepcional, lo cual provocó un crecimiento constante de la ciudad a lo largo del quinientos y seiscientos. Durante este tiempo, la ciudad de Cartagena llegó a ser cabeza de un hinterland propio en el que se había ido dibujando una

3. R. M. Hervás (1997); A. Martín García (2001); M. Gil Muñoz (2009), (2005); P. Ortega del Cerro (2018), (2019).

4. P. Herades (2017). 
sociedad prototípicamente estamental ${ }^{5}$. Si bien es cierto que no existió un fuerte grupo de nobleza titulada ni señorial, sí que se formó una oligarquía en base a hidalgos, labradores ricos y comerciantes. Al llegar el siglo XVIII, la ciudad era un núcleo urbano dentro de la Monarquía con recursos y con actividades relativamente diversas ${ }^{6}$.

Con la llegada del setecientos y el arribo de los Borbones al trono español, la marina de guerra es ampliamente reformada -el sistema de flotas y armadas de los Austrias es sustituido por la denominada Real Armada, un modelo centralizado y dirigido directamente desde la Secretaría de Estado y del Despacho de Marina- y Cartagena se convertirá en una de sus principales bases. En 1726 es nombrada capital del Departamento Marítimo del Mediterráneo y desde 1732 comienzan las obras del nuevo arsenal y astillero ${ }^{7}$. Además, hasta 1748 fue igualmente la sede de las galeras reales, única flota que no fue absorbida directamente por la Real Armada a comienzos de siglo. Todo ello hizo que la ciudad se convirtiera en un destacado centro de poder, polo industrial y nodo económico de demanda que fue parejo a un destacable aumento de población -en el censo de Godoy de 1797 se contabilizan 49.957, llegando al máximo en 1803, con 56.760 habitantes $-{ }^{8}$. La llegada de la oficialidad naval, aunque fue progresiva a lo largo de la primera mitad del siglo, tuvo como marco este crecimiento urbano en términos demográficos y económicos. Muy diferentes eran las situaciones de las otras dos grandes bases navales peninsulares; Cádiz era el puerto mercantil más importante de la Monarquía y cabeza del monopolio comercial con el imperio colonial, mientras que Ferrol era un pequeñísimo núcleo que apenas había sido refundado a comienzos de siglo.

Un primer retrato de la oficialidad naval en Cartagena lo podemos encontrar en el Catastro de la Ensenada, el cual nos descubre que en 1755 había en este puerto 79 oficiales del Cuerpo General de la Armada. Las diferencias entre ellos eran muy considerables ${ }^{9}$. En lo alto de la jerarquía se encontraba

5. R. Torres SÁnchez (1998).

6. V. Montojo (1993); V. Montojo y J. Hernández Franco (1996).

7. Hay una bibliografía bastante extensa, véase M. T. PÉrez-Crespo Muñoz (1992); J. P. MERINO (1981), 39-52.

8. Si en el siglo XVI la ciudad apenas contaba con 1.200 habitantes, a finales del siglo XVIII llegaría a los 50.000 habitantes, cifra similar a la Murcia, Zaragoza o Málaga del momento. Cádiz contaba con 70.000 como máximo y Madrid, como capital del reino, casi 170.000 .

9. Archivo General de la Región de Murcia, FR, 2.14.1. Documentos que son copias del Archivo General de Simancas (AGS), Dirección General de Rentas, 1. a remesa, leg. 1546. 
el Teniente General Blas de Barreda, de 60 años de edad, quien era además Comandante General del Departamento y, por tanto, una de las personalidades más importantes de la ciudad. Del generalato de la Armada también eran Agustín Idiáquez, Isidro Postigo y Juan de Lángara, aunque igualmente se pueden destacar a los capitanes de navío Pedro Justiniani y Felipe de Borja. Todos ellos, debido a su estatus social y su posición en la jerarquía naval, tuvieron un hogar donde acogían a otros familiares y numerosos criados. El mencionado Blas de Barreda, que era soltero, vivía con un mayordomo, un ayudante de cámara, un escribiente, un paje, un repostero, un cocinero con su ayudante, un cochero mayor, un cochero segundo y tres esclavos. El capitán de navío Felipe de Borja, en cambio, era viudo, tenía dos hijos - uno presbítero y otro teniente de navío-, tres hijas, una hermana viuda, así como dos criados y dos criadas. En el lado contrario nos encontraríamos a Francisco de Rojas Contreras, alférez de fragata de veinticuatro años y soltero.

La posición privilegiada de la oficialidad naval es incuestionable, tanto por razones de estatus social como por la importancia de la institución naval en la ciudad, aunque eso no es sinónimo de plena integración en la sociedad local. Es más, la documentación parece mostrar que la inserción social de los oficiales fue limitada en las primeras décadas del siglo, aunque fue adquiriendo progresiva fuerza en la segunda mitad. Dentro de este periodo, se pueden establecer dos periodos diferenciados, tomando las décadas de 1770 y 1780 como separación. Antes de estas fechas, se pueden apreciar unos niveles de rigidez social en las relaciones bastante más acusados, sobre todo cuanto más alta era la graduación militar. Por ejemplo, el marqués de Spínola, que era brigadier en el Departamento de Cartagena, hacía un testamento en 1777 donde dejaba como únicos albaceas al también brigadier de la Armada Alfonso Alburquerque, al coronel e ingeniero Mateo Vodopich -que era el director de las obras del arsenal- y a Gerónimo Cambino, capitán de lenguas de Su Majestad ${ }^{10}$. Por su parte, el Capitán General del Departamento de la ciudad en 1773, Carlos Reggio, hizo un testamento donde estipulaba algunos legados a su familia, pero dejó por comisarios y albaceas a Juan Domingo Medina, Intendente General de Marina en Cartagena, y al muy reverendo padre fray Diego Molina, guardián del convento de San Francisco de la ciudad ${ }^{11}$.

10. Archivo Histórico Provincial de Murcia (AHPMU), Cartagena, protocolo 6068, fols. 172-174.

11. Archivo Naval de Cartagena (ANCT), Testamentaría, caja 2415; AHPMU, Cartagena, protocolo 6064 , fols. $305-308$. 
Durante las décadas de 1750,1760 y 1770 hubo muestras de integración social, aunque una parte de los oficiales seguía constituyendo un grupo relativamente cerrado, tal y como pasaba en Ferrol. Un segmento de los cuadros medios de la oficialidad naval de estas décadas se relacionó esencialmente con militares de graduación similar y, muy secundariamente, con vecinos de la ciudad de un estatus social parecido. Por ejemplo, el capitán de fragata y caballero de Santiago Lorenzo Alderete instituía como único albacea en 1744 al capitán de alto abordo Rodrigo de Urrutia ${ }^{12}$. Por su parte, el capitán de navío Gerónimo Cabeza Tinoco decía en 1759 que quería que sus albaceas fueran su mujer, el capitán de fragata y director de pilotos Antonio Gregorio y el teniente de navío Antonio Parearroyo ${ }^{13}$.

Otra parte de la oficialidad emprendió un proceso de plena inserción en la sociedad local vía matrimonio. A finales de la década de 1750 se manda a la Secretaría de Estado de Marina una "relación de los oficiales que se han casado en Cartagena, después de que llegó la escuadra a este puerto, según se ha podido inquirir"14. Este documento, que ofrece datos de un periodo comprendido entre 1747 y 1758, señala que fueron 22 los oficiales casados en Cartagena, 21 con licencia y 1 sin ella. La mayoría de estos matrimonios se hizo con hijas de regidores del concejo -algunas parentelas lo hacen sistemáticamente- y de "caballeros particulares de la ciudad", esto es, familias distinguidas de la ciudad. También hubo enlaces matrimoniales con hijas de "comerciantes de puerta cerrada", es decir, al por mayor, y oficiales y empleados de las galeras de Cartagena.

Al casar con la élite local y el estrato mercantil superior de la ciudad, la oficialidad naval estaba retroalimentando su posición privilegiada en la estructura social. Es más, hay evidencias suficientes de que ciertas familias decidieron asentarse en esta ciudad y establecer aquí su base social. Un ejemplo de ello fue una de las ramas de los Ussel de Guimbarda. Familia de origen francés, estuvo durante el siglo XVII y XVIII en varias ciudades andaluzas -Málaga, Córdoba, Cádiz-, donde consiguieron integrarse en oligarquías locales ${ }^{15}$. Joaquín Ussel de Guimbarda, que era hijo del malagueño marqués de Ussel, llegó a Cartagena como oficial de la Armada y allí casó con Juana Negrete, proveniente de una familia recientemente asentada en la ciudad que se mudó para ejercer el servicio al rey en el ramo de la

12. AHPMU, Cartagena, protocolo 5727, fols. 300-302.

13. AHPMU, Cartagena, protocolo 5760, fols. 274-277.

14. AGS, Secretaría de Marina, leg. 13.

15. E. Soria Mesa (2000), 17; F. Andújar (2004), 251.

(C) Baetica. Estudios Historia Moderna y Contemporánea, 39, 2019, 321-348. Facultad de Filosofia y Letras, Universidad de Málaga. Departamento de Historia Moderna y Contemporánea 
administración. En 1748, aunque estuviera destinado temporalmente en Cádiz, instituía como albacea a su esposa y a Alonso Hernández de Villalba, regidor perpetuo de Cartagena ${ }^{16}$. En otros casos, como el de Francisco del Río Otero, que era teniente de fragata, las relaciones forjadas se dirigieron a las familias autóctonas y tradicionales de Cartagena, como los Martínez Fortún y los Agüera -ambas parentelas de labradores ricos y distinguidos del campo de Cartagena y de la urbe-, concretamente con Pablo Martínez Fortún y Francisco Agüera ${ }^{17}$. Muy parecidos fueron los albaceas de Mauro Sequeira, quien instituyó al mismo Francisco Agüera, alférez de fragata y piloto, al capellán José Martínez Fortún y a José Oraguren, tesorero de víveres de la Armada ${ }^{18}$.

\section{CONSOLIDACIÓN Y PLENITUD DE LA OFICIALIDAD EN LA CARTAGENA DEL SETECIENTOS}

A partir de la década de 1770 se pueden apreciar dos grandes pautas de comportamiento dentro de la oficialidad naval. Por un lado, un sector que, a través de sus enlaces matrimoniales con los miembros de la oligarquía local, estableció una verdadera simbiosis entre la élite de la ciudad, la sociedad cartagenera y el estamento militar destinado en Cartagena. A finales de la centuria, ciertas familias eran al mismo tiempo consolidadas sagas en el poder local y parte destacable del Cuerpo General de la Armada. Los apellidos Rato, Tacón, Rosique, Postigo, Tilly, Borja, Escaño, Valcárcel son algunos ejemplos. Por otro lado, encontramos a un grupo de la oficialidad naval que, sin establecer enlaces matrimoniales o vínculos familiares, se insertaron dentro de la cotidianeidad social de la ciudad. Sea un caso u otro, es evidente que durante el último cuarto del siglo XVIII y los primeros años del XIX, la oficialidad naval constituía un grupo esencial de la estructura social cartagenera.

Los oficiales que se integraron dentro de la élite local, del mismo modo que la parte de la oligarquía que ingresó en el Cuerpo General de la Armada, siguieron una estrategia bastante clara. El primer paso, sin lugar a dudas, era el matrimonio. A los oficiales les era ventajoso un casamiento con las hijas de los miembros de la élite local, al igual que la oligarquía veía con

16. AHPC, Cádiz, protocolo 1840, fols. 27-34.

17. AHPMU, Cartagena, protocolo 6060, fols. 58-60.

18. AHPMU, Cartagena, protocolo 5762, fols. 404-406. 
buenos ojos esos enlaces para mejorar el poder y el estatus familiar ${ }^{19}$. Como consecuencia de ello, algunos oficiales llegaron a convertirse en regidores de la ciudad, aunque no siempre como propietarios -usualmente, el suegro o la esposa cedían el cargo-, y formaron parte de la toma de decisiones de la política local. Póngase como ejemplo representativo a los hermanos Antonio, Joaquín, José, Martín y Teodoro Escaño García de Cáceres, todos ellos oficiales navales y entre los que destaca el general Antonio Escaño, quien será regente del Reino durante la Guerra de Independencia. Su padre, que era capitán de galeras y natural de Córdoba, se convirtió en alcalde perpetuo y noble de Cartagena gracias a su matrimonio con María Cristina García de Cáceres Garro, procedente de una de las principales familias de la oligarquía cartagenera desde el siglo XVI -eran poseedores de varias regidurías y sendos mayorazgos-.

Otro resorte de esta integración de la oficialidad en la élite local fue pertenecer a la Cofradía del Cristo del Socorro, cuya denominación completa era la Muy Devota, Venerable e Ilustrísima Cofradía de la Hermandad de Caballeros del Santísimo y Real Cristo del Socorro. Se podría incluso afirmar que esta era una prolongación del concejo, ya que se repiten exactamente los mismos nombres y apellidos ${ }^{20}$. La importancia de esta cofradía radica en que fue la única en toda la ciudad que exigía pruebas de nobleza y limpieza de sangre para ingresar. Había sido fundada por el duque de Veragua en 1691, cuando este era capitán de galeras en Cartagena, lo cual proporcionaba una aureola de distinción sin parangón en la ciudad. Los apellidos García Postigo, Tacón, Rosique, García de Cáceres, Borja, Valcárcel o Salafranca, entre otros, eran muestra de ese estrato superior de la sociedad cartagenera que emergió de la fusión de la oligarquía local y la oficialidad naval ${ }^{21}$. Ilustrativo es que 10 de los 29 hermanos existentes en 1819 eran militares; la amplia mayoría pertenecientes a las parentelas que se conjugaban entre la élite local y la Armada ${ }^{22}$.

19. F. Maestre de San Juan (2011); V. Montojo y J. Hernández Franco (2001); Tornel (2001).

20. En términos comparativos con Ferrol, Alfredo Martín ha estudiado la presencia de los oficiales en cofradías y asociaciones religiosas de este puerto gallego, en donde destaca la orden de San Francisco o la Hermandad de la Caridad. A. Martín García (2018).

21. Archivo Municipal de Cartagena (AMCT), CH 311, exp. 12.

22. AMCT, FO19017, exp. 4. No obstante, también hubo oficiales que, sin emparentar con la oligarquía, consiguieron entrar en la cofradía, tal como Sebastián Feringán o José Ambulodi. 
Un ejemplo singular y representativo de esta simbiosis fue Francisco Javier Everardo Tilly (1712-1795), quien llegaría a ser Capitán General de la Armada y recibió el marquesado de Casa Tilly en 1761. Este oficial, a pesar de los múltiples destinos que tuvo alrededor del mundo, decidió asentarse en Cartagena y llegaría a convertirse en una de las figuras más poderosas y relevantes de la ciudad ${ }^{23}$. Fue probablemente el oficial naval que más se enriqueció durante todo el setecientos -seguramente a través de negocios e inversiones en Indias-, y acumuló un patrimonio muy destacable ${ }^{24}$. A su llegada a Cartagena, en la década de 1740, casó con Teresa Panés González de la Reguera, única hija de Fulgencio Panés, regidor perpetuo del concejo y alguacil mayor del Santo Oficio, además de poseedor de un mayorazgo y conde de Pozo Nuevo ${ }^{25}$. Gracias a su suegro, Francisco Javier consiguió ocupar una regiduría del concejo cartagenero. Años más tarde, una vez que había quedado viudo, casó con Ana Llamas Mena, viuda de Alonso Oviedo, regidor de Cartagena y coronel de milicias ${ }^{26}$.

De su primer matrimonio tuvo dos hijas. María Pascuala Everardo Tilly y Panés, condesa de Pozo Nuevo, marquesa de Casa Tilly y Dama Noble de María Luisa, casó en Cartagena con Francisco de Borja y Poyo, un oficial de la Armada que había nacido en esta misma ciudad -hijo del capitán de navío y galeras Felipe de Borja y Tacón Garro de Cáceres, a su vez parte de las familias oligárquicas de la ciudad-, llegó a la cúspide de la jerarquía naval y recibió de su padre el marquesado de Camachos ${ }^{27}$. Se convirtió en gobernador político y militar de Cartagena entre 1806 y 1807. La otra hija, María Francisca Everardo Tilly y Panés, casó en 1770 en Cartagena con Pedro Rosique y González de Rivera, teniente de navío

23. Archivo Histórico Provincial de Cádiz (AHPC), San Fernando, protocolo 49, fols. 326-363; protocolo 52, fols. 249-254; protocolo 66, fols. 195-242

24. Según sus testamentos, numerosísimas piezas de tierra en el campo de Cartagena, varias haciendas, una casa principal en Cartagena, siete casas en Cartagena, un oficio de procurador en el ayuntamiento (lo ejercía por arriendo Agustín Molina), un oficio de escribano (arriendo Juan Orozco), una casa en Alguazas, baúles repletos de bienes y varias inversiones hechas en el comercio de Cádiz.

25. La familia Panés, de origen italiano, ya estaba asentada en Cartagena a finales del siglo XVII. Se enriqueció con el comercio, fundó varios mayorazgos de relativa importancia y accedió al concejo. F. Maestre de San Juan (2011), 108-109.

26. Según su testamento, era "hija legítima del Exmo. Sr. D. José de Llamas, marqués de Menahermosa, teniente general que fue de los Reales Ejércitos, natural de Rioja, reino de Granada y la Exma. Sra. Dña. Ana Magdalena de Mena, marquesa de Menahermosa, su mujer". AHPC, San Fernando, protocolo 72, fols. 286-293.

27. AHPMU, Cartagena, protocolos 6579 y 6581. 
de la Armada, regidor de Cartagena, alguacil mayor del Santo Oficio, caballero de la Orden de Santiago y caballero de la Maestranza de Valencia, pero sobre todo miembro de dos de las familias más importantes de la oligarquía local -los Tacón y los González de Rivera-.

Si todos estos datos son sintomáticos de la simbiosis que hubo entre la élite local y la oficialidad naval, cabe señalar, además, que el marqués de Casa Tilly construyó el que fue posiblemente el palacio más importante de la Cartagena del XVIII. Situado en la calle Mayor, la principal avenida de la ciudad, por la cual pasaba casi obligatoriamente toda la población, construyó un impresionante edificio sobre el antiguo palacio que perteneció al conde de Fernán Núñez - capitán general de galeras destinado en Cartagena- y otros edificios anexos. El palacio estaba presidido por una imponente portada de mármol negro coronado con un escudo de mármol blanco con las armas del marquesado.

La documentación notarial también resulta interesante para observar no solo el grado de inserción de la oficialidad naval en la sociedad cartagenera, sino también vislumbrar las formas que esta adoptó. Analizando las referencias que hicieron los oficiales en los protocolos, podemos afirmar que a partir de las décadas de 1770 y 1780 la integración del grupo fue mayor. Tómese como dato indicativo que el 58\% de los oficiales mostró relaciones con vecinos y naturales de Cartagena. En esta documentación también se pueden ver con claridad las dos grandes pautas de comportamiento en lo que se refiere a la inserción social. Por un lado, un conjunto de oficiales que fue estableciéndose poco a poco en este Departamento y convirtió a esta ciudad en su destino y su base familiar y social principal. Por otro lado, un grupo de oficiales que, aunque destinados en Cartagena, optaron por una inserción menos profunda, aunque eso no significa que no se relacionaran e integraran en la sociedad local a través de todo tipo de relaciones.

Como hemos mencionado, el primer grupo de oficiales consumó su unión con la ciudad a través de diversos enlaces matrimoniales que se produjeron entre las décadas de 1740 y 1780. La simbiosis entre oligarquía local y oficialidad naval fue tal que, a finales del siglo, había un número de familias que pueden ser consideradas tanto oligarquía local como élite naval: unas veces por locales que habían ingresado en la Armada-Tacón, Rosique, Rato, Anrich, García de Cáceres- y otras veces miembros de la élite naval que acabaron por incorporarse a la oligarquía -Tilly, García del Postigo, Borja, Baldasano, Escaño-. Véase como ejemplo al oficial Antonio Córdoba Romay, que era hijo del Capitán General y Director General 
de la Armada Luis de Córdoba. Este oficial se encontró durante muchos años destinado en Cartagena y allí hizo su último testamento -1782-. En él hacía constar que sus albaceas debían ser su suegra -este oficial se había casado en segundas nupcias con María Joaquina García del Postigo Poyo, procedente de una familia integrada tanto en la élite local como la oficialidad naval- y Pedro Rosique Rivera, miembro muy destacado de oligarquía cartagenera ${ }^{28}$.

Había otros oficiales que, sin haber enlazado matrimonialmente con la sociedad local, mostraron profusas relaciones con autóctonos y personas destinadas en Cartagena. Por ejemplo, el brigadier de la Armada Manuel Travieso nombraba como albaceas, entre otros, a: "Ignacio Madrid Jiménez, presbítero vicario de esta ciudad, comisario del Tribunal de la Inquisición; José Antonio Madrid Rosique, Escribano Mayor del Ayuntamiento; Leandro Echenique, oficial de la clase de primeros del Ministerio de Marina y secretario de la Comandancia General de este Departamento"29. Por su parte, el capitán de navío Francisco Javier Sarabia instituía a varios albaceas en 1787, la mayoría provenientes de la sociedad cartagenera: el religioso Juan de la Soledad; Francisco Rosique Gilabert, caballero de Santiago y Alguacil Mayor de la Inquisición; Teodoro Escaño, teniente de navío; y Justo Salafranca, sargento mayor de los Batallones de Marina ${ }^{30}$-aunque estos dos últimos eran militares, todos pertenecían a familias profundamente asentadas y consolidadas en el poder local-.

Del mismo modo, la relación con otros militares también fue destacable $-41 \%$ de los casos-, generalmente complementándose con las relaciones forjadas con los mencionados personajes de la sociedad local. No obstante, en otras ocasiones se optó por rodearse exclusivamente de oficiales, como ocurrió con el teniente de fragata Lorenzo Larrade en 1789. Este oficial nombró como albaceas a Alfonso Alburquerque y Juan de Aristizábal, capitán de navío y de fragata respectivamente. Por su parte, el alférez de navío Julián Power, que se encontraba soltero y era natural de Bilbao, nombró como albaceas a José Antonio Camino, teniente de navío, y José Tous, teniente de fragata ${ }^{31}$.

No podemos olvidar que la Cartagena del siglo XVIII, y especialmente durante la segunda mitad, se desarrolló a la sombra del arsenal y del Depar-

28. AHPMU, Cartagena, protocolo 6072, fols. 249-250.

29. AHPMU, Cartagena, protocolo 6073, fols. 68-70.

30. ANCT, Testamentaría, caja 2426, exp. 19.

31. AHPMU, Cartagena, protocolo 6079, s. f. y fols. 174-176. 
tamento, con todo lo que eso llevaba parejo -astilleros, refuerzos militares, industrias, aumento del comercio-. Es por ello que, durante el último cuarto del siglo, también se atestiguan relaciones con otros servidores de la Monarquía que estuvieron destinados en Cartagena -aparecen en el $30 \%$ de los casos-, aunque en campos civiles de la burocracia, administración y, en menor medida, la justicia. Eran individuos con los que, frecuentemente, trabajaban de manera estrecha y, además, compartían multitud de características sociales. Por ejemplo, el Teniente General de la Armada Adrián Cuadrón Cantein, máximo jefe militar del Departamento, nombraba como único albacea a Pedro Barrientos Rato, Intendente General de Marina, responsable jefe del ramo administrativo de la Armada en Cartagena ${ }^{32}$. Algo parecido, aunque en un estrato inferior de la jerarquía naval, ocurrió con José María Santa Cruz, alférez de navío. Este nombró como albacea a Antonio Vegue, oficial segundo del Ministerio de Marina, y como albacea y único heredero a Manuel Busta, contador de navío ${ }^{33}$. Por último, cabría citar a José Agustín Olózaga, quien en 1800 nombraba como albaceas a su esposa, a un religioso y a Genaro José Martínez, auditor honorario de Marina y Fiscal General ${ }^{34}$.

Saliendo del plano de las relaciones, no se puede acabar el análisis de este periodo sin señalar el papel que tuvo la oficialidad en la creación y desarrollo de la prensa local, lo cual es un perfecto indicador de la importancia que tuvo este grupo en la vida social de la ciudad. El Semanario Literario y Curioso de Cartagena fue una publicación que funcionó entre 1786 y 1788 . Tal y como señala en su primer número, nació de las entrañas de la Armada - desde la Imprenta del Departamento de Marina de Pedro Jiménez e impulsado por un grupo de oficiales, principalmente el capitán de navío Pedro Leiva- y su público principal, aunque no el único, fue la oficialidad naval - 'otras varias curiosidades, y noticias, cuya amenidad de varia lectura sirviendo de atractivo a los estudiosos de cualquier facultad, carrera u oficio, facilite al mismo tiempo los medios de una instrucción e ilustración general" - ${ }^{35}$.

Al publicarse la lista de suscriptores, es muy interesante observar cómo la oficialidad naval constituyó, sin duda, el grupo más numeroso

32. AHPMU, Cartagena, protocolo 6085, fols. 190-194.

33. AHPMU, Cartagena, protocolo 6079, fols. 488-489.

34. AHPMU, Cartagena, protocolo 6579, fols. 103-104.

35. M. R. García Hurtado (2010), (2004); J. M. Rubio Paredes (2004). 
-aproximadamente un tercio de total ${ }^{36}$-, del mismo modo que se puede examinar qué otros grupos estuvieron interesados en esta publicación $\mathrm{y}$, seguramente, fueron parte de los entornos sociales de los oficiales -otros militares, funcionarios de Marina, religiosos, comerciantes al por mayor ${ }^{37}$. Además, este documento también nos ofrece un panorama de los eventos y actos sociales principales de la ciudad, a los cuales la oficialidad podía y debía asistir. Destacan las celebraciones religiosas, tal como las novenas, predicaciones, misas en honor a vírgenes, misas solmenes, aunque hubo también actuaciones musicales y representaciones teatrales: "la compañía italiana sigue todas las tardes, excepto lunes y viernes, sus funciones de música y baile a las seis y media de la tarde. El domingo 3 del corriente representarán los aficionados en el Barrio de Santa Lucía extra-muros de esta ciudad la comedia el Jenizaro de Hungría, a las 4 de la tarde".

36. Los oficiales suscriptores eran tanto los que habían enraizado en la ciudad como aquellos que estaban destinados en Cartagena, pero no habían emparentado con familias de naturales. Algunos nombres son indicativos de ello, a saber: Andrés Tacón, caballero de Carlos III y capitán de navío; José Lorenzo Goycoechea, de la orden de San Luis y capitán de navío; Pedro Leiva, capitán de navío; Francisco Aguirre, capitán de navío; Francisco Ramos, teniente de navío; Domingo Tristán, guardiamarina; Juan Doral, teniente de navío; Guillermo Tournel, teniente de fragata, Martín Fernández de Navarrete, alférez de fragata y caballero de San Juan, Arcadio Pineda, alférez de navío; Tomás Naval, alférez de navío; Francisco Ruiz Cárdenas, capitán de navío; Francisco Trujillo, alférez de navío; Joaquín Vila, teniente de navío; Vicente Perler, teniente de navío; José Bonanza, capitán de navío; Miguel Tacón, capitán de navío; Juan Antonio Salinas, capitán de fragata; Ramón Deslobes, alférez de navío; José Meléndez, teniente de fragata; Martín de Olavide, alférez de fragata; Félix Marquina, capitán de navío; Ignacio Duque, brigadier; José Borja Tilly, alférez de fragata; Félix Estrada, capitán de fragata, Anotnio Ansoategui, capitán de navío; Ignacio Marrón, teniente de navío; Antonio García Portigo, capitán de fragata; Félix Melilupi, caballero de San Juan y capitán de fragata; Gernónimo Ballé, alférez de fragata; Ventura Barcaistegui, teniente de fragata; Francisco Castro, teniente de navío; Nicolás de Rojas, caballero de Santiago y capitán de navío; José Agustín Olózaga, capitán de fragata; Juan Francisco Pintado, teniente de fragata; Pedro Regalado, capitán de fragata; Ignacio Vago, alférez de navío; Juan Piña, capitán de fragata; Juan José García, capitán de fragata; José Barrientos, capitán de fragata; Félix Oneille, teniente de navío y caballero de Santiago.

37. Aunque la lista es muy amplia, se pueden citar los primeros suscriptores no militares: la viuda de Isaura e hijos, del comercio; Juan José Fardet, del comercio; Dr. Pedro Claver, médico del Real Hospital Militar de Marina; Felipe Cazorla, presbítero; José Alcaraz, presbítero; Francisco Rondón, guarda-almacén de la provisión de utensilios del reino de Murcia; Baltasar Rebuffat, con compañía de comercio; Patricio Núñez, comendador del convento de La Merced; Antonio Carreras, procurador; Baltasar Castillini, cónsul de Nápoles, etc. 


\section{LOS OFICIALES ANTE LA CRISIS DEL PRIMER TERCIO DEL SIGLO XIX}

Queda patente que la oficialidad naval constituía un grupo privilegiado dentro de la sociedad cartagenera, tanto en términos sociales como de poder. Póngase como ejemplo que, desde 1770 hasta 1820, la mayoría de los gobernadores políticos y militares de la plaza -cargo que sustituía al corregidor en determinadas ciudades- fueron oficiales de la Armada ${ }^{38}$. Esta relevancia se acentuó aún más durante la guerra de Independencia, momento en el que Cartagena se convierte en un nodo esencial para la resistencia contra los franceses -aunque el reino de Murcia fue invadido en 1812, la plaza portuaria resistió el asedio- ${ }^{39}$. Durante este periodo, un nutrido grupo de oficiales acaparó la defensa de la ciudad y fueron protagonistas de una cotidianeidad dominada por el contexto bélico ${ }^{40}$. Nada más tener noticias de los sucesos de mayo de 1808 se formó en la ciudad una junta compuesta por militares del Ejército y Marina, se proclamó la fidelidad al rey Fernando VII y se tomaron las primeras medidas para acondicionar la ciudad ${ }^{41}$. En 1809 se publicaba el "Plan general de defensa que dispone la Junta de Gobierno de esta plaza, para en caso de ser atacada por el enemigo", y en él queda constancia que tanto la oficialidad naval como el cuerpo de artillería del Ejército tendrían la batuta de las operaciones militares que hubiera que librar ${ }^{42}$. Los momentos de mayor tensión se produjeron en 1812, cuando prácticamente todo el reino de Murcia fue ocupado por los franceses, a excepción de Cartagena. En este momento toda la maquinaria defensiva se puso en marcha, consiguiendo que las tropas francesas se retiraran ${ }^{43}$.

38. Usualmente, aunque no siempre, compartiendo el cargo de Capitanes Generales del Departamento Marítimo. El teniente general de la Armada Carlos Reggio (1760-1773), el jefe de escuadra José Rojas (1773-1790), el jefe de escuadra Alfonso Alburquerque (1790-1794), el jefe de escuadra José Basco Vargas (1794-1796), el capitán general Francisco de Borja Poyo (1806-1807), el jefe de escuadra Gabriel Ciscar (1809-1810), el jefe de escuadra Francisco Javier Uriarte (1811-1812), el jefe de escuadra José Serrano (1812-1814) y el jefe de escuadra Juan de Dios Topete (1814-1822).

39. Sobre la guerra de Independencia en la ciudad de Cartagena hay varios trabajos, y todos ellos destacan el papel de la oficialidad naval. C. Tornel (2004); R. Frase (2006); P. M. Egea Bruno (1991); J. Gómez Vizcaíno (2008), (2007); C. Roda Alcantud (2009).

40. J. J. Sánchez Baena e A. Irigoyen López (2017); J. J. Sánchez Baena y L. Bouzón Calvo (2008); C. Roda Alcantud (2011).

41. AMCT, CH, 2294.

42. АМСТ, $\mathrm{CH}, 46$.

43. AMCT, LH, 308.

(C) Baetica. Estudios Historia Moderna y Contemporánea, 39, 2019, 321-348. Facultad de Filosofía y Letras, Universidad de Málaga. Departamento de Historia Moderna y Contemporánea 
Si bien es cierto que la contienda llevó a la oficialidad naval a un punto de máximo protagonismo en la ciudad, la Marina quedaría muy dañada y desde entonces comenzará una crisis que se agudizará durante las décadas de 1810 y 1820 . Vázquez de Figueroa, ministro de Marina, llegó a afirmar que: "Cádiz, Ferrol, Cartagena, puntos admirados en otro tiempo por sus soberbios y suntuosos Arsenales (...) en donde España representaba una idea exacta de su poder y su riqueza, son actualmente en donde con propiedad puede afirmarse que la cruel desolación y la espantosa miseria han fijado su lúgubre domicilio" 44 . También es ilustrativa la imagen que ofreció el viajero Mordecai en los años 1813 y 1814 cuando visitó la ciudad: "siempre ha sido una gran base naval y los almacenes, talleres y otros edificios necesarios en un Arsenal son numerosos y valiosos. Una buena escuela naval y Observatorio, frente al Puerto, y construido en el más simple y austero estilo arquitectónico, contribuyen al embellecimiento del lugar. Las calles son bastante más anchas que en otras ciudades y están muy sucias. Sus habitantes, desde la epidemia [de 1804], que casi anualmente se repite, junto a la decadencia de la potencia naval española, se han visto reducidos de 60.000 a 35.000 y son todos pobres. La ciudad tiene un aire triste y decadente" ${ }^{45}$. Esta oscura imagen que ofrece este viajero tendió, incluso, a recrudecerse en las décadas de 1820 y 1830.

La Cartagena de la segunda mitad del siglo XVIII y comienzos del XIX no se podía entender sin su fuerte impronta militar; la ciudad era, ante todo, una de las principales bases de la Armada, y gran parte de la urbe se movía en torno a este eje. Esto explica que, al igual que en San Fernando o Ferrol, cuando la Marina entró en crisis, la ciudad comenzó un proceso de decadencia. La oficialidad naval que quedó en la ciudad tuvo que pasar las penurias provocadas por la contienda, aunque además había que sumar los problemas internos de la Marina, tal como el retraso de las pagas de hasta 24 meses. El contexto social, político y económico de la ciudad, y de España en general, era totalmente distinto al de la segunda mitad del setecientos, lo que provocó que los oficiales tuvieran que adaptarse a las nuevas circunstancias. Al cambiar la estructura y orden social de la ciudad, la oficialidad naval también experimentó transformaciones en su inserción social. Si observamos las relaciones, se podrá comprobar que el vínculo

44. Exposición sobre el estado de la Marina Expuesta por el ministro Vázquez Figueroa, Madrid, 20 de octubre de 1812, citado en C. Roda Alcantud (2008), 8.

45. Mordecai M. NoAh, Travels in England, France, Spain, and the Barbary States in the years 1813-14 and 15, recogido en C. TORRES-Fontes (1996), 614. 
de los oficiales de la Armada con la élite local se fue difuminando, al tiempo que las antiguas familias de la oligarquía también experimentaron un periodo difícil. Póngase como ejemplo ilustrativo de esta época que en 1821 la mencionada Cofradía del Cristo del Socorro, espacio esencial para la sociabilidad de algunos oficiales de la Armada durante el XVIII, desapareció por falta de hermanos.

En lugar de la oligarquía y los estratos más altos, ahora los entornos sociales básicos de la oficialidad naval se compondrán de los grupos medios y medios-altos de la sociedad que durante este periodo comienzan a emerger ${ }^{46}$. Buena muestra de esta nueva realidad social naciente fue la propuesta de nuevos regidores de 1833, una vez planteada la enajenación de dichos cargos del concejo. Según Pedro M. Egea Bruno, el concejo se formaría de: tres profesores de Farmacia y Medicina, dos Abogado de los Reales Consejos, siete hacendados, cuatro labradores, tres escribanos, un oficial de Marina, dos comisarios de guerra, un procurador, un empleado de Reales Provisiones y nueve comerciantes. Se trata de un periodo en el que la oficialidad naval experimentó progresivos cambios en su forma de relacionarse e integrarse socialmente. Un ejemplo de ello lo ofrecen los comerciantes. Mientras que en el siglo XVIII la vinculación entre oficiales y comerciantes fue secundaria, en el ochocientos sí que encontramos lazos mucho más fluidos ${ }^{47}$. Un ejemplo es el teniente de navío Manuel Vera Rocafull, proveniente de una de las familias oligárquicas más antiguas de Lorca, y el comerciante Joaquín Fernández. El primero nombra a Joaquín como único albacea de su testamento en $1817^{48}$. También resulta significativo el caso del capitán de navío Pedro Alejandro Trogmontón Trujillo. En 1813 este oficial establecía como primer albacea a su esposa y, en caso de indisposición de esta, a dos sobrinos suyos y a Agustín Carlos Roca, a la sazón "escribano principal de Marina" en Cartagena ${ }^{49}$. Este ejemplo, aunque particular, es representativo de una nueva época. Los notarios, que conformaron un grupo social intermedio durante el siglo XVIII y tuvieron vetado el acceso a los estratos más altos, aparecen en el XIX como un emergente segmento de la

46. P. M. Egea Bruno (1996), 362.

47. El comercio ejercido en Cartagena, y por tanto sus comerciantes, era de un carácter secundario, de tipo local y provincial que distaba mucho de los niveles y el poder del comercio indiano gaditano. Buena muestra de ello es que después de la liberalización del comercio con Indias, solo un navío fue enviado a América. C. PArrón (1990).

48. AHPMU, Cartagena, protocolo 6601, fols. 687-688.

49. ANCT, Testamentaría, caja 2458, exp. 4. 
sociedad $^{50}$. Por tanto, que un oficial de la Armada mostrara explícitamente su plena confianza y amistad con un escribano puede entenderse como un indicador de nuevos patrones de relación.

El rasgo más significativo de este periodo en las relaciones sociales de la oficialidad naval en Cartagena fue el repliegue que se produjo dentro del propio cuerpo. Si durante las últimas décadas del siglo XVIII el $41 \%$ de los entornos sociales se componía de, al menos, un oficial de la Armada, la proporción en la primera mitad del XIX se incrementa hasta los dos tercios. El teniente general y capitán general del apostadero de Cartagena Baltasar Hidalgo de Cisneros protocoliza su testamento en 1830 resolviendo que sus albaceas debían ser: su esposa, el jefe de escuadra Marcelo Spínola y el capitán de navío Juan María Osorio ${ }^{51}$. Por su parte, el capitán de navío Antonio Barrientos Rato nombró en 1820 como albacea a sus hijos, a su sobrino y, junto a ellos, al capitán de navío Eduardo Bryant y al mencionado Baltasar Hidalgo de Cisneros $^{52}$. En ese mismo año, el teniente general de la Armada José Adorno daba su total confianza a "Vicente Ignacio Imperial Digueri, brigadier de los Reales Ejércitos, Francisco Millau, brigadier de la Armada, y Bernardino Rolandy, cura párroco de la Iglesia" ${ }^{53}$. También hubo casos de estrecha relación con el cuerpo del ministerio de la Armada -la rama administrativa de la institución-, como hizo el brigadier de la Armada Diego Alesón en 1834, quien depositó en su hijo y en Bernardo Abad, comisario de guerra del ministerio político de Marina, el poder de actuar como albaceas tras su muerte ${ }^{54}$.

Se percibe, por tanto, que la inserción social de la oficialidad naval durante el primer tercio del siglo XIX estuvo sujeta a dos tendencias que, aparentemente, son contrapuestas: por un lado, un relajamiento de las antiguas barreras y pautas de sociabilidad, entablando vinculación con grupos que habían sido claramente inferiores en tiempos anteriores; y por otro, un cierre en torno al cuerpo, lo cual les garantizaba cierta exclusividad y distinción en un tiempo en los que los esquemas sociales seguían unas tendencias inciertas. Este último hecho se atestigua igualmente en la presencia pública de los oficiales en eventos sociales de la ciudad. Por ejemplo, en 1820 se promulga la celebración "nacional" de los acontecimientos del 2 de mayo

50. M. A. Extremera (2009).

51. ANCT, Testamentaría, caja 2470, exp.3.

52. AHPMU, Cartagena, protocolo 6603, fols. 566-567.

53. Ibidem, fols. 512-515.

54. ANCT, Testamentaría, caja 2471, exp. 9. 
de 1808 , y en ella los oficiales debían ocupar una posición prioritaria ${ }^{55}$. No obstante, la presencia de militares en actos públicos fue un tema que requirió una regulación particular, y en 1821 se recupera un decreto de 1813 en el que se acuerda que "no deben los militares ir en cuerpo a funciones públicas, y si lo hacen como particulares convidados por las autoridades políticas, ocuparán el distinguido lugar que estas les designen"56.

\section{EL CUERPO GENERAL DE LA ARMADA EN LA SOCIEDAD CARTAGENERA DECIMONÓNICA}

A partir del segundo tercio del XIX, y sobre todo desde mediados de esta centuria, la ciudad de Cartagena experimentará una recuperación bastante destacable, llegando a ser a finales de siglo una urbe ciertamente grande y dinámica, relevante en términos sociales y económicos. Es importante señalar que durante el ochocientos la impronta militar y naval de la ciudad seguirá siendo muy importante, aunque no será el elemento único y central que la vertebre. Durante la década de 1840, la Marina de guerra volverá a ser potenciada a través de diversos planes navales, lo cual repercutió favorablemente sobre Cartagena. El vacío arsenal, los pobres soldados y los ruinosos almacenes volverán a recuperar cierto dinamismo, sobre todo a partir de la década de 1850, cuando se acometió un ambicioso programa de reforma del arsenal ${ }^{57}$. No obstante, a pesar de este resurgir de la Armada, la ciudad estará lejos de ver el trasiego naval del setecientos.

La recuperación económica de la ciudad y su comarca durante el XIX vino esencialmente de la activación de la minería. Se volverán a abrir las minas de plata, famosas desde la época romana, pero sobre todo se desarrolló la extracción de plomo y zinc ${ }^{58}$. De estos dos ejes -potenciación de la Marina y activación de la minería- se desencadenó un crecimiento económico que incluyó la instalación de industrias, la atracción de mano de obra y servicios, el aumento de la demanda, el incremento del comercio, etc. Es en este momento cuando verdaderamente se desarrolla el puerto civil de la ciudad, que hasta ese momento había sido secundario, convirtiéndose Cartagena en un importante enclave mercantil del Mediterráneo.

55. ANCT, Régimen General, III, h, 201.

56. ANCT, Régimen General, III, c, 194.

57. C. Roda Alcantud (2008b).

58. J. B. Vilar y P. M. Egea Bruno (1982), (1985). 
Además, en 1865 llega a la ciudad el ferrocarril a través de una línea directa con Madrid -pasando por Murcia y Albacete-. Todo ello se trasluce en la población de la ciudad y en el dinamismo social de la misma. En 1842 había 33.593 habitantes, pero en 1857 llegó a los 59.618, aumento que se mantendrá hasta alcanzar los 103.373 en 1900.

En paralelo, se produce una reestructuración social. Una nueva jerarquización, diferentes patrones de relación y nuevos espacios de sociabilidad emergen y se consolidan. Ejemplo de ello es que el Casino de Cartagena, espacio de sociabilidad por antonomasia de las élites decimonónicas, aparece en torno a 1836, y en 1833 se había fundado de Real Sociedad Económica de Amigos del País de Cartagena. Cabe, entonces, preguntarse qué papel jugó la oficialidad naval en este resurgir de la ciudad. Tal y como hemos indicado, la Marina siguió ocupando un lugar muy importante en la estructuración de la ciudad -además del arsenal, estaba el astillero, que constituía un nodo industrial de primer orden-, aunque perdió ese protagonismo absoluto y dominante que tuvo durante el XVIII.

Esta misma premisa parece que se adapta a la forma en que la oficialidad naval se insertó en la sociedad local; siguió constituyendo un grupo relevante, distinguido y en cierto modo privilegiado en la ciudad, pero no fue protagonista. Es más, se puede incluso afirmar que, pese a la importancia de la oficialidad, mantuvo una actitud bastante discreta y reservada, cuando no relegada, en muchas esferas de la vida social. Limitaron su campo de actuación al ámbito castrense y desarrollaron cierta distancia simbólica con otros grupos sociales y profesionales. Los miembros del Cuerpo General se replegaron socialmente sobre el mismo grupo y su participación pública se limitó a actos religiosos y celebraciones organizadas por la propia Armada. No obstante, este comportamiento no debe extrañar, pues fue durante la segunda mitad del XIX cuando se desarrolló con más fuerza un corporativismo en la sociedad española, y máxime en una ciudad de ferviente presencia obrera ${ }^{59}$.

Durante toda la segunda mitad del siglo XIX, la corporación local invitaba a la oficialidad de la Armada a tener representación en la procesión del Corpus Christi. El alcalde exhortaba anualmente a "los señores jefes y oficiales de los cuerpos de la Marina residentes en el departamento", aunque parece que la oficialidad no era especialmente receptiva. Por esta razón la propia Armada hacía listados de los oficiales que habían sido nombrados para asistir obligatoriamente a la procesión del Corpus. Por ejemplo, en 
1879 debían ir los capitanes de navío de segunda clase D. Eduardo Álvarez Estrada y Campos y D. Luis Gázquez Doral, el capitán de fragata D. José Ángel Muñoz Gionzález, los tenientes de navíos de primera clase D. José Pilón Sterling y D. Manuel MacCrohon Seidel, además de otros dos tenientes de navío de segunda clase y dos alféreces de navío ${ }^{60}$.

Finalmente solo asistieron tres de los nueve convocados -justificaron su ausencia por indisposición o motivos de salud-, lo cual demuestra la apatía por estos actos. Exactamente igual ocurría en la celebración de la Semana Santa y las eucaristías. En 1895 solo fueron los capitanes fragata Antonio Caro y Federico Fernández de Parga, una representación realmente pobre para lo que era el Cuerpo General. En 1897, la Capitanía General solo pudo obligar a asistir al director de la Escuela de Torpedos, un capitán de navío y el comandante de la provincia marítima. En cualquier caso, la Armada insistía en la presencia de estos oficiales para mantener la distinción simbólica del cuerpo dentro de la vida social ${ }^{61}$. Tal es la actitud de los oficiales que en 1906 se dictan "reglas sobre la obligación de asistir a los actos religiosos los jefes y oficiales (...) y prohíbe el ostentar sobre el uniforme distintivo alguno que no esté autorizado por reglamento".

Un comportamiento bien distinto se percibe en las celebraciones organizadas por la propia Marina por motivos castrenses o relacionados con el cuerpo. Por ejemplo, en 1866 la ciudad recibe la visita de un buque de guerra de los Estados Unidos. Los oficiales fueron los encargados de preparar su recibimiento, estancia, ocio y obsequios, pero en la orden se estipula "si bien es cierto es que voluntad de SM que los buques (...) sean atendidos (...) como puede presentarse aisladamente alguno de poco porte mandando por jefe de corta graduación, podrá en tal caso prescindirse de la reunión de sociedad y limitarse VE a dar a su comandante y oficiales un convite a que invitará a las autoridades superiores de la provincia y cónsul de su nación" 62 .

60. ANCT, Régimen General, III, g, 200. Es llamativo observar que ese año no asistieron Eduardo Álvarez Estrada, por "encontrarse algo indispuesto", Luis Gázquez, porque no tenía el uniforme preparado, José Ángel Muñoz, José Milón, Manuel MacCrohon y Rodolfo Matz, todos ellos por no encontrarse en buen estado de salud.

61. Ibidem.

62. ANCT, Régimen General, III, h, 201. Se trata de la contestación a una orden anterior que decía: "La reina se ha servido disponer que en caso de presentarse en este puerto algún buque de guerra de los Estados Unidos, aparte de tratar a toda la dotación con la más exquisita deferencia, con arreglo a los principios de cortesía que se establece en el derecho de gentes entre naciones amigas, sean además obsequiados su coman- 
Una actitud mucho más proactiva se encuentra en actos que repercuten sobre el propio cuerpo y la Marina. Por ejemplo, en 1895 se celebró el entierro del contralmirante Juan Flores Prichard y se movilizó "al mayor número posible de oficiales", mientras que en 1891 ocurrió algo parecido cuando llegó a la ciudad el cadáver del teniente general José de los Reyes $\mathrm{Mesa}^{63}$. Pero especialmente destacable es la propuesta que se hizo en 1882 por "varios jefes y oficiales de este departamento" para la "celebración de un aniversario en conmemoración de las glorias tradicionales del Cuerpo General de la Armada" ${ }^{64}$. La idea parece que surgió en el brindis del banquete posterior a una misa en honor a todos los oficiales navales muertos en batalla -"por la patria, por el rey, por la Marina, por la íntima unión del Cuerpo General de la Armada y por el engrandecimiento y brillo de la escuadra nacional" se decía en la propuesta-.

Este repliegue social al ámbito castrense también se puede apreciar en los protocolos notariales. Si analizamos estos documentos, se puede comprobar la presencia bastante frecuente de clérigos, de otros militares y de funcionarios del cuerpo de intendencia de la Marina. Por ejemplo, el teniente de navío Pedro Villar López, que era soltero, protocoliza su testamento en 1853 y deja como albaceas a Juan Espín, oficial tercero del Cuerpo del Ministerio de la Armada, y a una mujer llamada Magdalena, "que vive en su casa y compañía" ${ }^{5}$. El contralmirante Mariano Fernández Alarcón Bilbao nombraba en 1870 como albaceas, en igualdad de poderes, al brigadier José Baldasano, a su mujer y a su hijo Mariano ${ }^{66}$. Pero estas muestras no significan que los oficiales no tuvieran relaciones con otros grupos sociales.

En 1841 el capitán de fragata Joaquín Bilbao Angosto protocolizaba su testamento declarando que había casado con Ana García del Postigo, perteneciente a una de esas familias que encarnó la simbiosis entre élite naval y oligarquía local en el setecientos, y había tenido cuatro hijos. Su citada esposa había muerto y, ante las contingencias de la vida, puso especial énfasis en el devenir de sus hijos. Pidió a su "amigo" Antonio Gómez Segura que "no abandone a sus hijos" y nombraba como tutores

dante y oficiales con un convite y reunión de sociedad, a fin de corresponder de que nuestra marina es objeto en aquella nación y a la noble conducta que su gobierno está observando".

63. ANCT, Régimen General, III, 1, 202.

64. ANCT, Régimen General, IX, c, 347.

65. AHPMU, Cartagena, protocolo 6616, fols. 156-158.

66. AHPMU, Cartagena, protocolo 11614, fols. 271-275. 
y curadores de sus vástagos a Andrés Gabarrón, Francisco Barry y Antonio Murcia $^{67}$. El primero llegaría a ser regidor de la ciudad durante el Bienio Progresista (1854-1856 ${ }^{68}$ y, según Pedro M. Egea, formaba parte de "una burguesía conceptualmente como urbana, integrada por abogados, catedráticos, notarios, médicos, boticarios y arquitectos, que apenas superaba el $5 \% "$ "69.

El brigadier de la Armada Alejandro Silva realizaba su testamento en el año 1868 y nombraba por albaceas a su sobrino Leandro Alesón Millau y a sus dos "buenos amigos", como él mismo denominaba, Fulgencio Teruel y el ya citado Antonio Murcia ${ }^{70}$. El primero era abogado de la ciudad -fue también nombrado auditor interino de Marina- y se había convertido en regidor durante el reinado de Isabel II, aunque también participó en la creación de una compañía mercantil con la importante familia de los Dorda - una de las principales familias burguesas y mineras de la ciudad de la segunda mitad del XIX-71.

Cabe citar finalmente otro elemento que puede ser interesante para comprender la inserción social de la oficialidad en Cartagena. Aunque hay registros muy escasos, se ha podido comprobar que una parte de la oficialidad se mostró activa en ese dinámico ciclo de la economía de Cartagena a través de diversas inversiones, lo cual supone otra faceta de la integración en la ciudad. Por ejemplo, el jefe de escuadra José Montojo Albizu declaraba en 1866 que había entregado 2.290 pesos a los hermanos Bosques, del comercio de Cartagena, para que produjeran ciertas rentas que tendrían por objetivo proporcionar recursos y educación a un pequeño niño que este general había adoptado ${ }^{72}$. En cambio, el almirante Pascual Cervera Topete dejó constancia de su participación en la Compañía Cartagenera de Navegación -creada en 1900, aunque comenzó a operar en 1915, por iniciativa de importantes comerciantes de la ciudad- a través de cuarenta acciones por valor de 20.000 pesetas y treinta obligaciones que sumaban 15.000 pesetas $^{73}$.

También se puede mencionar la minería, aunque la variable y diversa capacidad económica de los oficiales de la Armada hizo que su partici-

67. ANCT, Testamentaría, caja 2474, exp. 4.

68. M. Ríos SanMartín (2002), 11.

69. P. M. Egea Bruno (1987-1989), 1581.

70. AHPMU, Cartagena, protocolo 11614, fols. 62-66.

71. V. Montojo (2016), 97-117; J. M. Rubio Paredes (2005), 5

72. AHPC, Cádiz, protocolo 111, fols. 849-882.

73. AHPC, Medina Sidonia, protocolo 8252, fols. 656-694. 
pación tuviera amplios contrastes. Algunos de ellos hicieron inversiones relativamente pequeñas, como el brigadier de la Armada Pedro Pilón. En 1865 dejaba constancia de que tenía "algunas" acciones en La Encarnación y Legos Francos en Cartagena, ambas sociedades mineras ${ }^{74}$. En 1882 el oficial Ramón Fossi Bish decía que tenía dos acciones de minas en Cartagena de la sociedad Los Amigos ${ }^{75}$. Por su parte, el brigadier de la Armada Alejandro Silva decía en 1870 que tenía una acción en la sociedad cartagenera que explotaba la mina Mesías en la sierra de Almagrera; media acción en la sociedad Unión, dueña de la mina titulada Apostolado, de la misma sierra; y otra acción de la sociedad que explotaba la mina Julia de la sierra de Cartagena ${ }^{76}$.

\section{CONCLUSIONES}

Este trabajo tenía como objetivo analizar la forma en que la oficialidad naval se insertó socialmente en Cartagena, ciudad donde se asentó una de las bases de la Armada española. Se quería conocer cuál fue el papel de este grupo dentro de la sociedad local, cuáles fueron los grupos con los que tuvieron relación los oficiales y cómo era su presencia en la vida social. La principal conclusión que se obtiene del examen realizado es que la integración de la oficialidad dependió en gran medida de la posición y relevancia que tenía la Marina dentro de la urbe.

A comienzos del siglo XVIII se creó el Departamento Marítimo de Cartagena, y desde entonces la ciudad se convirtió en una base naval de primer orden. Se construyó el arsenal y los astilleros, además de otros edificios -Capitanía General, Hospital Militar, Cuartel de Artillería, Cuartel de Infantería, Academia de Guardias Marinas, nuevas murallas, Cuartel de Presidiarios y Esclavos-, de tal modo que la Armada se convirtió en el motor y eje estructurante de la ciudad, sobre todo durante la segunda mitad de la centuria. La oficialidad naval adquirió una posición correlativa a este hecho y se convirtió en uno de los grupos más distinguidos de la estructura social.

Además de ser el cuerpo rector de la institución naval, un número destacable de oficiales emprendió un profundo proceso de integración en la élite local a través de matrimonios, la ocupación de regidurías o el ingreso en

74. AHPC, Cádiz, protocolo 545, fols. 3446-3455.

75. AHPC, San Fernando, protocolo 509, fols. 2071-2074.

76. AHPMU, Cartagena, protocolo 11614, fols. 62-66. 
la cofradía del Socorro. En cualquier caso, la totalidad de la oficialidad de esta época se codeaba con los estratos más altos de la sociedad, tal como la oligarquía y nobles locales, otros oficiales del Ejército, funcionarios reales o comerciantes al por mayor. La relevante posición de los oficiales queda constatada en el hecho de que ellos fueron los promotores de la primera publicación periódica de la ciudad, pensada precisamente para todos esos sectores sociales con los que se relacionaban.

El protagonismo de la oficialidad naval en la vida local llegó a su máximo exponente durante la guerra de la Independencia. El contexto bélico explica que estos militares acapararan no solo los principales puestos de poder, sino que fueran los protagonistas de una cotidianeidad marcada por la defensa de la base naval y la plaza fuerte. Esta situación cambió radicalmente una vez acabada la contienda. La profunda decadencia de la Marina sumergió a la ciudad en una aguda crisis. Al depender la urbe de los fondos destinados a la Armada y toda la actividad que ella generaba, se produjo un colapso social y económico. Consecuentemente, la oficialidad perdió su protagonismo, al tiempo que se experimentaba un relajamiento de los patrones sociales establecidos hasta ese momento.

A partir de 1850 se abre una nueva etapa; la reactivación de la Marina, el desarrollo de la minería y el aumento de comercio hizo que la ciudad recuperara dinamismo social y económico, aunque la oficialidad, aún siendo un grupo destacado y privilegiado, optó por una posición ciertamente secundaria en la vida social. Si bien es cierto que fueron los oficiales parte importante de la cotidianeidad, se produjo un cierre sobre el propio cuerpo, lo cual seguramente les garantizó cierto grado de exclusividad y distinción. Desde entonces, la oficialidad parece conformar un verdadero estamento castrense dentro de la ciudad que, sin estar alejado de la sociedad local, deseó mantener distancia y rasgos distintivos.

\section{BIBLIOGRAFÍA}

Andújar Castillo, Francisco (2004), El sonido del dinero: monarquía, ejército y venalidad en la España del siglo XVIII, Marcial Pons, Madrid.

Egea Bruno, Pedro María (1987-1989), "Bases sociales y económicas del liberalismo cartagenero (1833-1868)”, Estudios Románicos, 6, pp. 1579-1592.

- (1991). "Cartagena durante la Guerra de la Independencia: la actuación de la Junta de Gobierno (1808-1811)", Agua, s.p.

- (1996), "Los siglos XIX y XX", en C. Tornel Cobacho (coord.), Manual de Historia de Cartagena, Ayuntamiento de Cartagena y Universidad de Murcia, Murcia, pp. 350-372. 
Extremera, Miguel Ángel (1990), El notariado en la España Moderna. Los escribanos públicos de Córdoba (siglos XVI-XIX), Calambur, Madrid.

FrASER, Ronald (2006), La maldita guerra de España. Historia social de la guerra de la Independencia, 1808-1814, Crítica, Barcelona.

García Garralón, Marta (2018), "La educación de los oficiales de la Armada en el siglo XVIII. La Real Academia de Guardias Marinas", en J. MARChEnA Fernández y J. Cuño Bonito (eds.), Vientos de guerra. Apogeo y crisis de la Real Armada, 1750-1823, vol. 1, Doce Calles, Madrid, pp. 121-327.

García Hurtado, Manuel Reyes (2006), "Un periódico para la Real Armada: el Semanario Literario y Curioso de la ciudad de Cartagena (1786-1788)", en M. Cantos Casenave (coord.), Redes y espacios de opinión pública: De la Ilustración al Romanticismo: Cádiz, América y Europa ante la Modernidad, 1750-1850, Universidad, Cádiz, pp. 89-104.

- (2010), "La participación de los militares españoles en la prensa del siglo XVIII", Studia Historica. Historia Moderna, 32, pp. 375-398.

Gil Muñoz, Margarita (2005), "Militares y marinos: morir en el Madrid de la Ilustración”, Madrid: Revista de Arte, Geografía e Historia, 7, pp. 269-302.

- (2009), "Marinos en el Madrid del siglo XVIII: entorno vital", Revista de Historia Naval, 27, pp. 39-70.

- (2015), La oficialidad de la Marina en el siglo XVIII. Un estudio sociológico (1700-1758), Ministerio de Defensa, Madrid.

Gómez Vizcaino, Juan Antonio (2007), "La defensa de la plaza de Cartagena durante la Guerra de la Independencia (1808-1814)", en Cartagena y su Departamento Marítimo en la guerra de la Independencia, Instituto de Estudio Carthaginenses, Cartagena, pp. 29-54.

- (2008), La guerra de la Independencia en Cartagena (1808-1814), Editorial Corbalán, Cartagena.

Herades Ruiz, Pedro José (2017), “El sistema mediterráneo hispánico y la base naval de Cartagena”, en M. P. Pi Corrales, J. Cantera Montenegro y E. Martínez Ruiz (coords.), Frontera y fortificación, Actas, Madrid, pp. 623-639.

Hervás Avilés, Rosa M. (1997), “Los marinos del rey: estirpe, linaje y parentesco de una élite", en J. CASey y J. Hernández Franco (eds.), Familia, parentesco y linaje: Congreso Internacional Historia de la Familia: Nuevas perspectivas sobre la sociedad europea, Universidad, Murcia, pp. 395-413.

Maestre de San Juan, Federico (2011), "La aristocracia de Cartagena en el siglo XVIII", Murgetana, 125, pp. 103-126.

Martín García, Alfredo (2001), "La oficialidad de la Armada en el Departamento de Ferrol, 1780-1857", Revista de Historia Naval, 75, pp. 67-82.

- (2017), "Expansión urbana y asociacionismo religioso en la Galicia de finales del Antiguo Régimen: cofradías, hermandades y órdenes terceras en la Real Villa de Ferrol”, Tiempos Modernos. Revista Electrónica de Historia Moderna, 36, pp. 534-558. 
Marinos en tierra: inserción social de la oficialidad naval en la ciudad...

Merino, José P. (1981), “Cartagena: el arsenal ilustrado del Mediterráneo español”, Áreas: Revista Internacional de Ciencias Sociales, 1, pp. 39-52.

Montojo, Vicente (1993), El siglo de oro en Cartagena (1480-1640): evolución económica y social de una ciudad portuaria del sureste español, Real Academia Alfonso X el Sabio, Murcia.

- (2016), "Fuentes para la Historia del comercio de Cartagena en los años 18651936", Murgetana, 134, pp. 97-117.

Montojo, Vicente y Hernández Franco, Juan (1993), "Cultura de honor, linajepatrón y movilidad social en Cartagena durante los siglos XVI y XVII”, Hispania, 185, pp. 1009-1030.

- (2001), "Patronazgo real y familiar urbanas: comportamientos de poder (Cartagena, siglo XVII-XVIII)”, en F. Chacón Jiménez y J. HernándeZ Franco (eds.), Familias, poderosos y oligarquías, Universidad, Murcia, pp. 81-92.

O’Donnell y Duque de Estrada, Hugo (2002), "La formación del oficial en el siglo XVIII. El marino ilustrado", en De la Paz de París a Trafalgar (17631805), Génesis de la España Contemporánea. VII Jornadas de Historia Militar, Ministerio de Defensa, Madrid, pp. 43-68.

Ortega del Cerro, Pablo (2019), "Redes de relación e integración social de los oficiales de la Armada en Ferrol, 1740-1870", Cuadernos de Estudios Gallegos, 132, pp. 285-312.

- (2018), “Aproximación a la inserción social de la oficialidad naval en el espacio gaditano, 1725-1900”, Memoria y Civilización. Anuario de Historia, 21, pp. 575-610.

PARrón SAlas, Carmen (1990), “Comercio marítimo y comerciantes en Cartagena en el siglo XVIII", Revista de Historia Naval, 29, pp. 23-62.

Pérez-Crespo Muñoz, María Teresa (1992), El arsenal de Cartagena en el siglo XVIII, Editorial Naval, Madrid.

Perfecto García, Miguel Ángel (2006), "El corporativismo en España: desde los orígenes a la década de 1930", Pasado y Memoria. Revista de Historia Contemporánea, 5, pp. 185-218.

Ríos Sanmartín, Miguel Á. (2002), “El Bienio Progresista en Cartagena (18541856), Cartagena Histórica, 11, pp. 9-15.

Roda Alcantud, Cristina (2008a), "La guerra de la Independencia en Cartagena y su departamento marítimo", en E. De Diego y J. L. Martínez SAnz (eds.), El comienzo de la guerra de la Independencia, Madrid, Actas, p. 6.

- (2008b), Historia e ingeniería en el siglo XIX. Vanguardia de la industria naval en el Mediterráneo Occidental: el arsenal de Cartagena, Ayuntamiento y Universidad Politécnica, Cartagena.

- (2008) "Fuentes para el estudio de la historia naval: los fondos del Archivo General Intermedio de la Armada en Cartagena", en E. Nicolás Martín y González Martínez, C. (eds), Ayeres en discusión: temas clave de Historia Contemporánea hoy, Universidad, Murcia, s. p. 
- (2011), "El final del Antiguo Régimen y las primeras décadas de la España Liberal en los arsenales de la Península”, en J. J. SÁnchez Baena, C. Chaín Navarro y L. Martínez Solís (coords.), Estudios de Historia Naval: actitudes y medios en la Real Armada del siglo XVIII, Universidad, Murcia, pp. 91-124.

Rubio Paredes, José M. (2005), “El levantamiento progresista de 1844 en Cartagena”, Cartagena Histórica, 33, pp. 3-12.

Rubio Paredes, José M. (2004), El "Semanario literario y curioso de la ciudad de Cartagena" (1786-1788): ocio de oficiales de la Armada "ilustrados" destinados en su departamento, Real Academia Alfonso X el Sabio, Murcia.

Tornel Cobacho, Cayetano (coord.) (1996), Manual de Historia de Cartagena, Ayuntamiento de Cartagena y Universidad de Murcia, Murcia.

Tornel Cobacho, Cayetano (2001), El gobierno de Cartagena en el Antiguo Régimen, 1245-1812, Ayuntamiento de Cartagena y Real Academia Alfonso $\mathrm{X}$ el Sabio, Cartagena.

- (2004), Gobierno local y quiebra del Antiguo Régimen en Cartagena. Antecedentes (1808-1812), Real Academia de Alfonso X el Sabio, Murcia.

Torres-Fontes SuÁrez, Cristina (1996), Viajes de Extranjeros por el Reino de Murcia, tomo II, Real Academia Alfonso X el Sabio, Murcia.

Torres SÁnchez, Rafael (1998), Ciudad y población: el desarrollo demográfico de Cartagena durante la Edad Moderna, Real Academia Alfonso X el Sabio, Murcia.

Sánchez Baena, Juan José e Irigoyen López, Antonio (2017), “La Armada y la Iglesia en tiempos de Independencia: la Asociación de Jesús Sacramentado de Cartagena, 1808-1814", Hispania Sacra, 139, pp. 319-332.

Sánchez Baena, Juan José y Bouzón Calvo, Laura (2008), "La Academia de Guardiamarinas de Cartagena en el contexto de la invasión napoleónica y la pérdida de los territorios americanos (1808-1824)", en E. Nicolás Martín y González Martínez, C. (eds.), Ayeres en discusión: temas clave de Historia Contemporánea hoy, Universidad, Murcia, s. p.

Soria Mesa, Enrique (2000), El cambio inmóvil. Transformaciones y permanencias en una élite de poder (Córdoba, siglos XVI-XIX), Ayuntamiento, Córdoba.

Vilar, Juan Bautista y Egea Bruno, Pedro María (1982), "Minería y sociedad en el distrito de Cartagena durante el Sexenio Democrático (1868-1874)", Hispania, 152, pp. 607-654.

- (1985), La minería murciana contemporánea (1840-1930), Universidad de Murcia-Caja Murcia, Murcia. 